\title{
DISKUSSIONSFORUM
}

\section{Die Debatte über TA - ohne Ziel und deshalb endlos?}

Ein Kommentar zu A. Grunwalds Artikel: „Arbeitsteilige Technikgestaltung und verteilte Beratung: TA zwischen Politikberatung und Technikbewertung in Unternehmen" in TA-Datenbank-Nachrichten 2/2001

\section{von Dr. Martin Socher, Dresden}

\section{Einleitung}

Die aktuelle Debatte über Technikfolgenabschätzung führt weniger zu mehr Klarheit als vielmehr zu mehr Verunsicherung. Ein klares Ziel ist nicht erkennbar, die Akteure stellen neue Konzepte vor, ohne den klassischen TAAnsatz mit seinen anerkannten Stärken zu integrieren. Zukunftsfähige TA sollte sich auf ihre nachgewiesene analytische Stringenz, interdisziplinäre Arbeitsweise, Diskurs- und Ergebnisoffenheit gründen und sich einem breiten Markt für Beratungsleistungen öffnen. TA sollte Plattform für die Bewältigung komplexer transdisziplinärer Prozesse sein und ihren eigenen Anspruch als Instrument der Politikberatung reduzieren.

\section{Dynamik als Selbstzweck}

Die Technikfolgenabschätzung tut sich auch nach ihrer erfolgreichen Etablierung in Deutschland schwer mit sich selbst. Dies hat eine Reihe externer und interner Gründe, über die im Weiteren einige vorläufige Überlegungen angestellt werden sollen.

- Obwohl mittlerweile erfolgreich verdrängt, wirkt das Trauma der Auflösung des Office of Technology Assessment (OTA) des amerikanischen Kongresses als umlaufende Stoßwelle nach, hatte doch das OTA als Legitimation für Politikberatung auf höchstem fachlichen und politischen $\mathrm{Ni}$ veau gedient. Studien des OTA galten als Benchmarks für eigene Arbeiten und deren anerkannte Wirkung auf politische Meinungsbildungsprozesse als anzustre- bendes Ziel. Insbesondere der Funktion von TA als Instrument der Politikberatung wurde nachgeeifert und versucht, diese angemessen in den deutschen/europäischen Kontext umzusetzen. Das weitestgehend spurlose Verschwinden des OTA löste in der europäischen TA-Community eine tiefsitzende Sinnkrise aus, an deren Überwindung offensichtlich auch durch verstärkte Aktivitäten gearbeitet wird.

- In der externen Wahrnehmung stellt sich die TA-Community als äußerst heterogenes methodisches Gebilde dar - was sie im Übrigen auch sein will - und lässt damit ein wichtiges Verkaufsargument, den „unique selling point“" vermissen. Mittlerweile fällt es selbst gut informierten Anwendern von TA-Studien schwer, diese methodisch- konzeptionell zu verorten und damit ihre fachliche Solidität erfolgreich gegenüber Skeptikern zu verteid igen.

- Das ständige Nachgeben gegenüber externer Kritik durch (scheinbar?) neue methodische Ansätze führt zu einer gewissen Beliebigkeit, die von den Kritikern der TA als konzeptionelle Schwäche wahrgenommen wird. Nach außen projizierte Dynamik wird zum Selbstzweck.

\section{Selbstfindung als Notanker}

Würde man TA als die Technik der Folgenabschätzung und damit wieder als strategisches Rahmenkonzept betrachten (H. Paschen 1978 bis 1997), dann könnten ihre immanenten Stärken: analytische Stringenz, Interdisziplinarität, Diskurs- und Ergebnisoffenheit Grundlage ihrer Revitalisierung sein. Dazu bedarf es allerdings der internen Selbstfindung und des Ablassens von hart erarbeiteten und liebgewonnenen Errungenschaften. Dazu zählt insbesondere der Anspruch, TA hätte (zumindest im Ergebnis) immer auch der Politikberatung zu dienen. Diese hochhängenden Trauben sind sicherlich süß, mit abnehmender Erreichbarkeit erscheinen sie dann doch als sauer. Nicht jedes TA Projekt, das mit dem Anspruch der Politikberatung durchgeführt wurde, findet dort eine hinreichende Resonanz. Der Bedarf an umfassenden TA-Arbeiten wird von der Politik selbst definiert, deren Primat bestimmt auch die Wirksamkeit bzw. Wirkung von TA. Mit der 
Etablierung des Büros für TechnikfolgenAbschätzung beim Deutschen Bundestag (TAB) wurden sichtbar Anspruch, Umfang und intendierte Wirkung parlamentarischer TA definiert. Ein darüber hinausgehender Beratungsbedarf existiert zwar, wird offenbar jedoch über andere Strukturen und Formen in Anspruch genommen. Um das OTA-Syndrom nicht zu vertiefen, muss sich deshalb die TA noch weiter als bisher den Märkten für Beratungsdienstleistungen öffnen. Dies bedeutet, dass die Wirtschaft, Kirchen, Gewerkschaften, aber auch NGO's oder der generierende Wissenschaftsbetrieb als Klienten akzeptiert und offen umworben werden sollten. Dies wird nur dann erfolgreich sein, wenn der Nachfrager konkreter TA-Projekte davon überzeugt werden kann, dass methodisch - konzeptionelle Klarheit vorausgesetzt - seine Position prozessorientiert unterstützt wird, ungeachtet dessen, dass das Endprodukt durchaus im Gegensatz zu seiner ursprünglichen Erwartungshaltung stehen kann. Die Stärke der TA liegt m. E. auch zukünftig in ihrem prozessorientierten Charakter. Eine TA wird durchgeführt um ,herauszufinden, was passiert wenn...“, nicht um zu beweisen, dass jemand Recht hat. Mit diesem Anspruch könnte zu den authentischen Wurzeln der TA zurückgekehrt und gleichze itig ein neues Klientel erschlossen werden. Allerdings sollte TA nie ausschließlich nachfragegetrieben sein; die bereits erwähnten Stärken legen es nahe, inhaltliche Angebote zu formulieren, diese durch Grundlagenforschung $\mathrm{zu}$ substantiieren und zeitnah insbesondere mit der interessierten Öffentlichkeit darüber zu kommunizieren. Dafür bedarf es weiterhin der staatlichen Förderung und des Vorhaltens entsprechender Kapazitäten.

\section{TA ist keine Wissenschaft, sondern eine Methode}

Im Wissenschaftsbetrieb sprießende „Orchideenfächer" legen die Vermutung nahe, dass der damit einhergehende Differenzierungsprozess zur „Kannibalisierung “' der großen Wissenschaftsdisziplinen führt. Während sich z. B. die Physik bemüht, ihr theoretisches Gerüst auf eine einheitliche Grundlage zu bringen, versuchen eine Reihe neuer Subdisziplinen ihre Marktposition durch immer weiteres Abdriften von den als zu starr empfundenen Mutterdisziplinen zu verbessern. Hochspezialisierten Experten ausdifferenzierter Subdisziplinen fällt es schwer, transdisziplinär zu agieren. Dies ist insbesondere dann problematisch, wenn wissenschaftliche Erkenntnisse oder technische Entwicklungen zu gesellschaftlich relevanten Implikationen führen. Verordnete Transdisziplinarität oder Delegation der ethischen Probleme auf die Ebene der Spitzenfunktionäre der Wissenschaftsorganisationen löst dieses Dilemma nicht. Würde jedoch TA als die Technik der Folgenabschätzung definiert werden, dann böte es sich an, diese in breitem Umfang als methodisches Konzept und als prozessorientiertes Instrument der transdisziplinären Forschung und deren nachhaltiger Umsetzung in den wissenschaftlichen Mutterdisziplinen zu berücksichtigen. Da TA weder eine Wissenschaft noch eine abgeleitete Subdisziplin ist, steht sie somit auch nicht im Wettbewerb mit dem ,normalen“ Wissenschaftsbetrieb. Darin liegt die Chance, die weitere Ausdifferenzierung der TA- Landschaft und damit deren Kannibalisierung zu verhindern. Da der klassische Forschungsbetrieb selten interdisziplinär, geschweige denn transdisziplinär organisiert ist, bietet die TA eine geeignete Plattform für Akteure und Themen, um Zwänge und Hierarchien tradierter Strukturen aufzulösen und fachübergreifend Innovationsprozesse voranzubringen. Mit einem solchen - zugegeben minimalistischen - Ansatz könnte TA wieder an Attraktivität gewinnen und Beiträge zu einer nachhaltigen Entwicklung liefern.

\section{Anmerkung}

1) Kannibalisierung hat seine Ursprünge in der Wehrtechnik, noch funktionierende ,wehrtechnische Gegenstände“ werden ,ausgeschlachtet“, um andere Gegenstände ähnlicher Art funktionsfähig zu halten.

\section{Kontakt}

Dr. Martin Socher

Westendstr.10, 01187 Dresden

E-Mail: martin.socher@gmx.net 